\title{
Cardiología de la mujer: ja buena hora!
}

\author{
Women's cardiology: At good time!
}

\author{
Alejandra Ibáñez $z^{1,2}$ \\ ${ }^{1}$ Cañavera Ibañez Cardiólogos, Montería, Córdoba; ${ }^{2}$ Capítulo de la Mujer, Sociedad Colombiana de Cardiología y Cirugía Cardiovascular, Bogotá. Colombia.
}

¿Es el corazón de la mujer diferente al corazón del hombre? Sí, y en realidad este no es el problema; el problema es que muy pocos lo saben. Es precisamente este desconocimiento lo que ha movido al Capítulo "Cardiología de la Mujer" de la Sociedad Colombiana de Cardiología y Cirugía Cardiovascular a consolidar un espacio permanente en una revista tan prestigiosa en la comunidad científica nacional e internacional como lo es la Revista Colombiana de Cardiología. La falta de información y de formación en la cardiología de género ha llevado a la triste realidad con que nos enfrentamos hoy: las enfermedades cardiovasculares son la principal causa de muerte para las mujeres, especialmente la cardiopatía isquémica, que todavía se considera una enfermedad del hombre. Hoy las mujeres no están siendo conscientes de los riesgos asociados a la enfermedad cardiovascular, y gran parte de la responsabilidad de esta situación recae sobre los profesionales de la salud que no nos formamos en cardiología de género y, por ende, no actuamos en consecuencia. Si queremos mejorar la detección de las enfermedades cardiovasculares en la mujer tenemos que empezar también por exigir una mayor representación del sexo femenino en estudios y ensayos clínicos, que son la mayor fuente de información sobre la eficacia de estrategias terapéuticas. La mayor parte de la información que manejamos sobre la enfermedad cardiovascular de la mujer proviene de estudios en los que las mujeres representan menos del $30 \%$ de la población analizada. Es imperativa una mayor inclusión del género femenino en los estudios científicos o la elaboración de algunos en forma exclusiva a la población femenina. El desconocimiento en las diferentes formas de presentación de los síntomas isquémicos del corazón de la mujer deriva en un retraso en la sospecha de enfermedad cardiovascular y en la menor utilización de estudios complementarios; es decir, las mujeres estamos subdiagnosticadas. A esto se suma que recibimos menos o más tarde el tratamiento adecuado, es decir, subtratadas.

Hoy más que nunca necesitamos profesionales de la salud que entiendan la problemática de la enfermedad cardiovascular en la mujer y que la entiendan no sólo desde nuestras diferencias hormonales/biológicas, sino también desde los comportamientos, roles y estilos de vida que nos exige la sociedad moderna.

Este valioso espacio de la Cardiología de la Mujer dentro de la Revista Colombiana de Cardiología es para nuestro Capítulo de vital importancia porque permitirá mejorar la educación de la comunidad científica en cuanto a la enfermedad cardiovascular de la mujer y estimulará la investigación y su aplicación para hacerla efectiva y tangible.

El mayor conocimiento de la problemática multifactorial de la enfermedad cardiovascular de la mujer se traducirá en diagnóstico y tratamiento mejores y oportunos, y se reflejará en nuestra meta final: la disminución de la morbimortalidad de la enfermedad cardiovascular en la mujer.

\section{Correspondencia:}

${ }^{*}$ Alejandra Ibáñez

E-mail: mariaalejandraibanez@ hotmail.com
Disponible en internet: 19-03-2021 Rev Colomb Cardiol. 2021;28(1):1-2 www.rccardiologia.com 0120-5633 / @ 2020 Sociedad Colombiana de Cardiología y Cirugía Cardiovascular. Publicado por Permanyer. Este es un artículo open access bajo la licencia CC BY-NC-ND (http://creativecommons.org/licenses/by-nc-nd/4.0/). 
Rev Colomb Cardiol. 2021;28(1)

Queremos equidad de género a la hora de la prevención, equidad de género a la hora del diagnóstico, equidad de género a la hora del tratamiento, equidad de género a la hora de la rehabilitación y equidad de género a la hora de la representación en estudios científicos.

Por eso, hoy decimos: "Cardiología de la mujer: ja buena hora!" 